\title{
Fatores Relevantes na Avaliação Cognitiva da População Portuguesa
}

\section{Relevant Factors on Cognitive Evaluation of the Portuguese Population}

Catarina Resende de OLIVEIRA $\square^{1}$, Anabela MOTA-PINTO², Vítor RODRIGUES ${ }^{3}$, Catarina ALVES ${ }^{4}$ Acta Med Port 2017 Apr;30(4):293-301 - https://doi.org/10.20344/amp.7299

\section{RESUMO}

Introdução: O envelhecimento é um processo inevitável que tem um impacto social e será nas próximas décadas o grande desafio em termos de saúde pública. Um sistema de saúde eficiente requer uma reflexão sobre as medidas preventivas a aplicar.

Material e Métodos: A amostra em estudo englobou 2672 indivíduos de ambos os géneros, com idade igual ou superior a 55 anos, residentes em Portugal continental, ao qual foi aplicado um questionário que incluiu as seguintes secções: Rede social; Locomoção; Autonomia física; Autonomia instrumental; Avaliação cognitiva; Atividade física.

Resultados: O estudo do perfil de envelhecimento da população Portuguesa revelou que a autonomia física para tarefas relacionadas com o quotidiano, se associam a um melhor desempenho cognitivo. Verificámos uma associação estatisticamente significativa entre o desempenho na avaliação cognitiva e o género, a idade, a escolaridade, o facto de a pessoa viver sozinha, o número de horas que está sozinha, a autonomia a andar na rua, lavar-se, vestir-se, comer, preparar refeições, fazer compras, gerir dinheiro, tomar medicamentos e lavar/tratar a roupa.

Discussão: A avaliação cognitiva é influenciada negativamente pelo número de horas que um individuo vive sozinho.

Conclusão: As atividades da vida diária devem ser valorizadas, uma vez que requerem capacidade para planear e realizar tarefas e a sua preservação é uma componente chave num envelhecimento de sucesso.

Palavras-chave: Actividades da Vida Diária; Alterações da Cognição; Educação; Envelhecimento; Portugal

\section{ABSTRACT}

Introduction: Aging is an inevitable process that has a social impact in the forecoming decades, and it will present a great challenge regarding public health. An efficient health system requires a reflection on the preventive measures to be implemented.

Material and Methods: The study population comprised a total number of 2672 individuals of both genders, aged 55 years and over, residents in continental Portugal, to whom a questionnaire was applied that included the following sections: Social network; Locomotion; Physical autonomy; Instrumental autonomy; Cognitive assessment; Physical activity.

Results: The study of aging in the Portuguese population found that physical autonomy for tasks related to daily life are associated with better cognitive evaluation. A statistically significant association was found between performance in cognitive assessment and gender age, schooling, the fact of living alone, the number of hours being alone, autonomy to walk in the street, washing, dressing, eating, preparing meals, doing shopping, managing money and taking medications and washer / treat clothing.

Discussion: Cognitive evaluation is negatively influenced by the number of hours that an individual is alone.

Conclusion: Activities of daily life must be valued, since they require the ability to plan and carry out tasks and their preservation is a key component in successful aging.

Keywords: Activities of Daily Living; Aging; Cognition Disorders; Education

\section{INTRODUÇÃO}

O envelhecimento é um processo inevitável que tem um indiscutível impacto social. Será nas próximas décadas o grande desafio em termos de saúde pública. Prevê-se que durante os próximos cinco anos e pela primeira vez na história, as pessoas com idade igual ou superior a 65 anos superarão o número de crianças com menos de cinco anos de idade. Um quarto da população mundial, terá mais de 65 anos em 2020, e a faixa etária com idade igual ou superior a 85 anos é a que apresentará o maior crescimento. ${ }^{1}$ Esta mudança demográfica é um desafio na gestão e nas políticas de saúde, uma vez que o envelhecimento está associado a alterações fisiológicas e psicológicas do indivíduo. O desenvolvimento socioeconómico e os avanços na medicina, baixaram a mortalidade e morbilidade por doenças infeciosas, mas o estilo de vida e os fatores de risco daqui acrescidos, associados a uma maior longevidade e menor mortalidade, aumentaram a incidência e a prevalência das doenças crónicas. Nove em cada dez indivíduos com idade igual ou superior a 60 anos têm pelo menos um fator de risco cardiovascular, e se apresentarem dois ou mais destes fatores de risco, têm uma maior suscetibilidade para desenvolver um défice cognitivo, especialmente nos domínios da atenção e do controlo executivo. ${ }^{2}$

Os sistemas de saúde precisam de encontrar estratégias eficazes para adequar os cuidados de saúde às necessidades dos indivíduos com mais de 60 anos de idade. Um sistema de saúde eficiente, tendo em conta o envelhecimento populacional e a grande proporção da população

1. Instituto de Bioquímica. Faculdade de Medicina. Universidade de Coimbra. Coimbra. Portugal.

2. Instituto de Patologia Geral. Faculdade de Medicina. Universidade de Coimbra. Coimbra. Portugal.

3. Serviço de Higiene e Medicina Social. Faculdade de Medicina. Universidade de Coimbra. Coimbra. Portugal.

4. Eurotrials, Consultores Científicos. Lisboa. Portugal.

$\triangle$ Autor correspondente: Catarina Resende de Oliveira. catarina.n.oliveira@gmail.com

Recebido: 09 de dezembro de 2015 - Aceite: 23 de dezembro de 2016 | Copyright @ Ordem dos Médicos 2017 
mundial a viver com carências económicas, requer uma reflexão sobre as medidas preventivas a aplicar e os encargos financeiros associados, de modo a definir as metas a atingir em termos de saúde a nível global. A heterogeneidade das alterações funcionais das populações envelhecidas, reflete o efeito cumulativo das desigualdades em cuidados de saúde ao longo da vida. ${ }^{3}$

Há vários exemplos de envelhecimento bem-sucedido, tanto a nível cognitivo como físico e de procura de um estilo de vida ativo. Na última década, o esforço para retardar o declínio cognitivo tem sido alvo de uma nova abordagem em investigação, que demonstrou que várias atividades do quotidiano, como o exercício físico regular e a estimulação cognitiva, aumentam a plasticidade cerebral, mesmo numa idade avançada. ${ }^{4}$

O recurso a um questionário de atividades da vida diária (AVDs), é um método comum de avaliação do estado funcional do idoso, muitas vezes utilizado como indicador da função cognitiva, nomeadamente quando focado em atividades complexas..$^{5-7}$ Este questionário inclui o registo de um conjunto de atividades que habitualmente são realizadas sem dificuldade pelos idosos. As AVDs, podem ser agrupadas em 'AVDs básicas' (AVDsB) relacionadas com o autocuidado como lavar-se e vestir-se, e 'AVDs instrumentais' (AVDsI) relacionadas com atividades mais complexas tais como gerir dinheiro, fazer compras e tomar medicamentos. ${ }^{8}$

Tem-se assumido, que pelo menos quatro fatores podem desempenhar um papel fundamental na forma como o ser humano se adapta à idade: a interação social, a nutrição, a estimulação cognitiva e a atividade física., ${ }^{4,9}$

A solidão é uma queixa subjetiva que retrata um sentimento no qual a pessoa sente uma profunda sensação de isolamento. É comum entre as pessoas mais velhas e está intimamente ligada à diminuição do número de contatos sociais e ao consequente afastamento da rede social. Estes factos estão associados a um declínio do estado de saúde das populações mais idosas, e tem sido sugerido serem preditivos de um subsequente declínio funcional e aumento da mortalidade. Da mesma forma, o envelhecimento de sucesso está relacionado com a manutenção de uma rede social ativa. ${ }^{10}$

A análise dos benefícios do exercício físico e consequente melhoria da autonomia física e do estado cognitivo do individuo idoso, deve ser criteriosamente analisada devido à grande variabilidade e vaga definição de atividade física. ${ }^{11}$ Sabe-se que no envelhecimento, impedir a redução da massa muscular e o aumento da massa gorda, se acompanha de uma maior independência nas atividades da vida diária e de uma maior longevidade. ${ }^{12}$

O estudo do perfil de envelhecimento da população Portuguesa (EPEPP), teve como objetivo geral definir o perfil do envelhecimento da população portuguesa de forma a determinar as prevalências de dependências de terceiros e caracterizar factores funcionais. ${ }^{14,16-23}$ No âmbito deste estudo e como um dos objetivos específicos, realizaram-se análises das possíveis associações entre os estilos de vida e o declínio cognitivo, tendo em conta que ao definir grupos de risco, uma intervenção precoce pode ter impacto numa perspetiva de política de saúde pública.

Numa fase em que Portugal se encontra em crise económica, consideramos importante acautelar a possível influência de fatores que possam mais tarde condicionar o envelhecimento com qualidade. As condições de trabalho, o tipo de ocupação, as trajetórias de carreira, e a complexidade do trabalho, são todos fortes fatores preditores da função cognitiva. ${ }^{13}$

\section{MATERIAL E MÉTODOS \\ Amostra em estudo}

A população em estudo reuniu indivíduos de ambos os géneros, com idade igual ou superior a 55 anos, residentes em Portugal continental, cujo nome constava da lista telefónica nacional e que deram o seu consentimento informado por escrito (Declaração de Helsínquia, 2004). Este estudo foi aprovado pela Comissão de Ética da Faculdade de Medicina da Universidade de Coimbra. Os dados que pudessem permitir a identificação de qualquer participante foram codificados antes do manuseamento da informação e não foram registados ou tratados dados que permitissem a identificação pessoal.

Foram excluídos todos os indivíduos que não correspondiam a estes critérios de inclusão e que, por incapacidade que implicasse retenção no leito e/ou cadeira, não se pudessem deslocar ao local de inquérito, ou que por impossibilidade de resposta (escrita ou verbal) não pudessem responder ao inquérito. ${ }^{14}$

A dimensão da amostra foi calculada a partir de dados descritos em estudos anteriores tendo em conta a heterogeneidade de perfis tipo de envelhecimento. ${ }^{15,16} \mathrm{O}$ estudo englobou um número total de 2672 indivíduos com idade igual ou superior a 55 anos, representativa da população portuguesa, nomeadamente no que diz respeito ao grupo etário, género e zona geográfica, foi efectuada uma amostragem estratificada, tendencialmente proporcional, com base nos censos de 2001 Instituto Nacional de Estatística (INE). Com esta amostra o erro de precisão máximo nas estimativas de prevalências de interesse para o estudo foi de $2 \%$, com um intervalo de confiança de $95 \%$.

Da amostra total, 1000 indivíduos $(37,4 \%)$ pertencem ao grupo etário dos 55 aos 64 anos, 978 indivíduos (36,6\%) pertencem ao grupo etário dos 65 aos 74 anos e 694 indivíduos $(26,0 \%)$ pertencem ao grupo etário com 75 ou mais anos. Dos 2672 indivíduos inquiridos, 1533 são do sexo feminino $(57,4 \%)$ e 1139 do sexo masculino $(42,6 \%)$.

O questionário para recolha de informação foi validado pela implementação de um pré-teste aplicado a um número reduzido de indivíduos em concelhos rurais e urbanos, abrangendo os estratos etários selecionados de forma a aferir a inteligibilidade do questionário e a necessidade de eventuais modificações. O pré-teste foi repetido aos mesmos indivíduos pelos mesmos inquiridores, com o intervalo de um mês (método do duplo teste) e avaliado o coeficiente de concordância através do teste Kappa de Cohen para 
Tabela 1 - Score de avaliação cognitiva

\begin{tabular}{|c|c|c|}
\hline Parcelas do score & Score de avaliação cognitiva & Classes do score de avaliação cognitiva \\
\hline $\begin{array}{l}\mathrm{AC}=\mathrm{OT}_{\mathrm{q} 1}+\mathrm{OT}_{\mathrm{q} 2}+\mathrm{OT}_{\mathrm{q} 3}+\mathrm{OT}_{\mathrm{q} 4} \\
+\mathrm{OT}_{\mathrm{q} 5}+\mathrm{OE}_{\mathrm{q} 1}+\mathrm{OE}_{\mathrm{q} 2}+\mathrm{OE}_{\mathrm{q} 3} \\
+\mathrm{OE}_{\mathrm{q} 4}+\mathrm{OE}_{\mathrm{q} 5}+\operatorname{Ret}_{\mathrm{q} 1}+\operatorname{Ret}_{\mathrm{q} 2}+ \\
\mathrm{Ret}_{\mathrm{q} 3}+\mathrm{Ac}_{\mathrm{q} 1}+\mathrm{Ac}_{\mathrm{q} 2}+\mathrm{Ac}_{\mathrm{q} 3}+\mathrm{Ac}_{\mathrm{q} 4}+ \\
\mathrm{Ac}_{\mathrm{q} 5}+\mathrm{Ev}_{\mathrm{q} 1}+\mathrm{Ev}_{\mathrm{q} 2}+\mathrm{Ev}_{\mathrm{q} 3}+\mathrm{Nom}_{\mathrm{q} 1} \\
+\mathrm{Nom}_{\mathrm{q} 2}+\mathrm{Rep}_{\mathrm{q} 1}+\mathrm{Comp}_{\mathrm{q} 1}+ \\
\mathrm{Comp}_{\mathrm{q} 2}+\mathrm{Comp}_{\mathrm{q} 3}+\mathrm{Leit}_{\mathrm{q} 1}+\mathrm{Esc}_{\mathrm{q} 1} \\
+\operatorname{Des}_{\mathrm{q} 1}\end{array}$ & AC varia entre 0 e 30 & 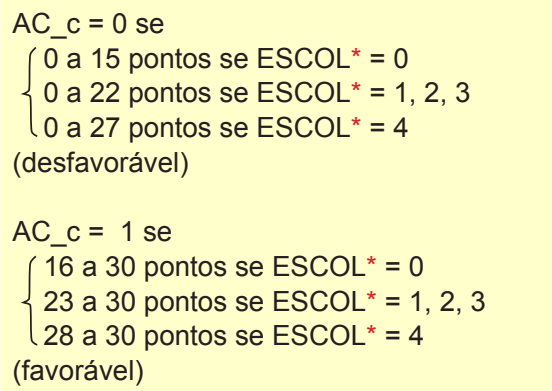 \\
\hline
\end{tabular}

variáveis categoriais. Os inquiridores foram selecionados e previamente formados pela Eurotrials.

A estrutura do questionário que se aplicou a esta análise, incluiu as seguintes secções: Rede social; Locomoção; Autonomia física; Autonomia instrumental; Avaliação cognitiva; Atividade física.

\section{Análise estatística}

Todas as análises estatísticas foram efetuadas através do software estatístico SPSS ${ }^{\circledR}$, versão 18.0 , tendo sido considerado o nível de significância de $5 \%$ em todas as análises inferenciais. Qualquer valor de probabilidade $p \leq 0,05$ representou o que se considera como diferença estatisticamente significativa nas análises comparativas entre grupos de interesse.

Foram definidos scores com base nas respostas às questões do inquérito, de acordo com o número de questões e tipo de questão. Foram considerados diferentes intervalos de variação para cada score, embora todos fossem codificados de 0 ou 1 , com 0 correspondendo à situação desfavorável e 1 à situação favorável.

\section{Análise descritiva}

Foi efetuada inicialmente uma análise descritiva univariada de todas as variáveis envolvidas: viver sozinho, horas sozinho por dia, ajuda para andar na rua, lavar-se, vestir-se, comer, preparar refeições, fazer compras, gerir dinheiro, tomar medicamentos, lavar/tratar a roupa e score de avaliação cognitiva favorável/desfavorável.

Foram criadas variáveis binárias referentes à ajuda para andar na rua, lavar-se, vestir-se, comer, preparar refeições, fazer compras, gerir dinheiro, tomar medicamentos e lavar/tratar a roupa foram recodificadas em variáveis binárias $(0 / 1)$ para casos autónomos e não autónomos.

\section{Análise inferencial}

Foram efetuadas análises comparativas do score de avaliação cognitiva favorável/desfavorável face às variáveis viver sozinho, horas sozinho por dia, ajuda para andar na rua, lavar-se, vestir-se, comer preparar refeições, fazer compras, gerir dinheiro, tomar medicamentos e lavar/tratar a roupa. Estas análises envolveram o teste do qui-quadrado.
Para as variáveis binárias: viver sozinho, horas sozinho por dia, autonomia a andar na rua, lavar-se, vestir-se, comer, preparar refeições, fazer compras, gerir dinheiro, tomar medicamentos e lavar/tratar a roupa foram também efetuadas análises comparativas utilizando o teste do qui-quadrado e calculados os respetivos odds ratio.

\section{Análise multivariada}

Foram calculados odds ratio ajustados e respetivos intervalos de confiança a 95\%, através da análise de regressão logística múltipla, para as variáveis que mostraram associação estatisticamente significativa com a avaliação cognitiva favorável/desfavorável na análise inferencial.

\section{Cálculo do score de autonomia física, autonomia ins- trumental e avaliação cognitiva}

Foi criada a variável score de autonomia física (AFs) de acordo com a soma das variáveis: lavar-se/tomar banho, vestir-se/despir-se, deitar-se/levantar-se, sentar-se/ levantar-se, utilizar sanitários, ajuda no descontrolo urina, ajuda no descontrolo fezes, comer/alimentar-se.

O score de autonomia instrumental teve em conta as variáveis: usar telefone, fazer compras, gerir o seu dinheiro, tomar medicamentos no último mês/ano, usar transportes, preparação das refeições, tarefas domésticas, lavar/ tratar da roupa.

A variável score de avaliação cognitiva (Sc-AC) foi calculada de acordo com a soma das variáveis: Orientação temporal (OT), Orientação espacial (OE), Retenção (Ret), Atenção e cálculo (Acl), Evocação (Ev), Nomeação (Nom), Repetição (Rep), Compreensão (Comp), Leitura (Leit), Escrita (Esc), Desenho (Des), assim como a variável score de avaliação cognitiva favorável/desfavorável (Ac-C) a partir dos critérios indicados na Tabela 1, tendo em conta a escolaridade (ESCOL).

A variável numérica 'escolaridade' (ESCOL), dividiu os anos de escolaridade completos em cinco níveis: $0=$ até três anos, $1=4$ a 6 anos, 2 = 7 a 9 anos, $3=10$ a 11 anos, 4 = a partir de 12 anos.

\section{RESULTADOS}

Análise comparativa de variáveis capazes de influenciar a avaliação cognitiva 
Foi efetuada uma análise comparativa através do teste do qui-quadrado para os resultados do score de avaliação cognitiva e as variáveis em estudo, tal como consta na Tabela 2 .

Observou-se uma associação estatisticamente significativa entre o género $(p<0,001)$, a idade $(p<0,001)$, a escolaridade $(p<0,001)$, viver sozinho ou acompanhado e a avaliação cognitiva $(p=0,001)$. Obteve-se uma observação semelhante quando se analisou a associação entre o número de horas que o indivíduo passa sozinho por dia e a avaliação cognitiva $(p=0,025)$.

Os indivíduos com menor autonomia para se lavarem apresentavam uma maior prevalência de score desfavorável, sendo essa associação estatisticamente significativa $(p<0,001)$. O mesmo valor de $p$ foi registado na associação entre score e grau de ajuda necessário para preparar refeições, fazer compras e gerir dinheiro, notando-se no geral maior prevalência de score desfavorável com maior necessidade de ajuda.

Observou-se também uma associação estatisticamente significativa entre o grau de ajuda para lavar/tratar a roupa e o score de avaliação cognitiva $(p=0,028)$. Registou-se o menor valor de prevalência de score desfavorável nos indivíduos que necessitavam de ajuda regular para manusear peças grandes (por exemplo, lençóis, toalhas de mesa $(3,8 \%)$.

Análise comparativa de variáveis capazes de influenciar a avaliação cognitiva com agregação de categorias

Após recodificação das variáveis não binárias em variáveis binárias, repetiu-se o teste do qui-quadrado e calculou-se o odds ratio e respetivos intervalos de confiança para

Tabela 2 - Análise comparativa de variáveis capazes de influenciar a avaliação cognitiva

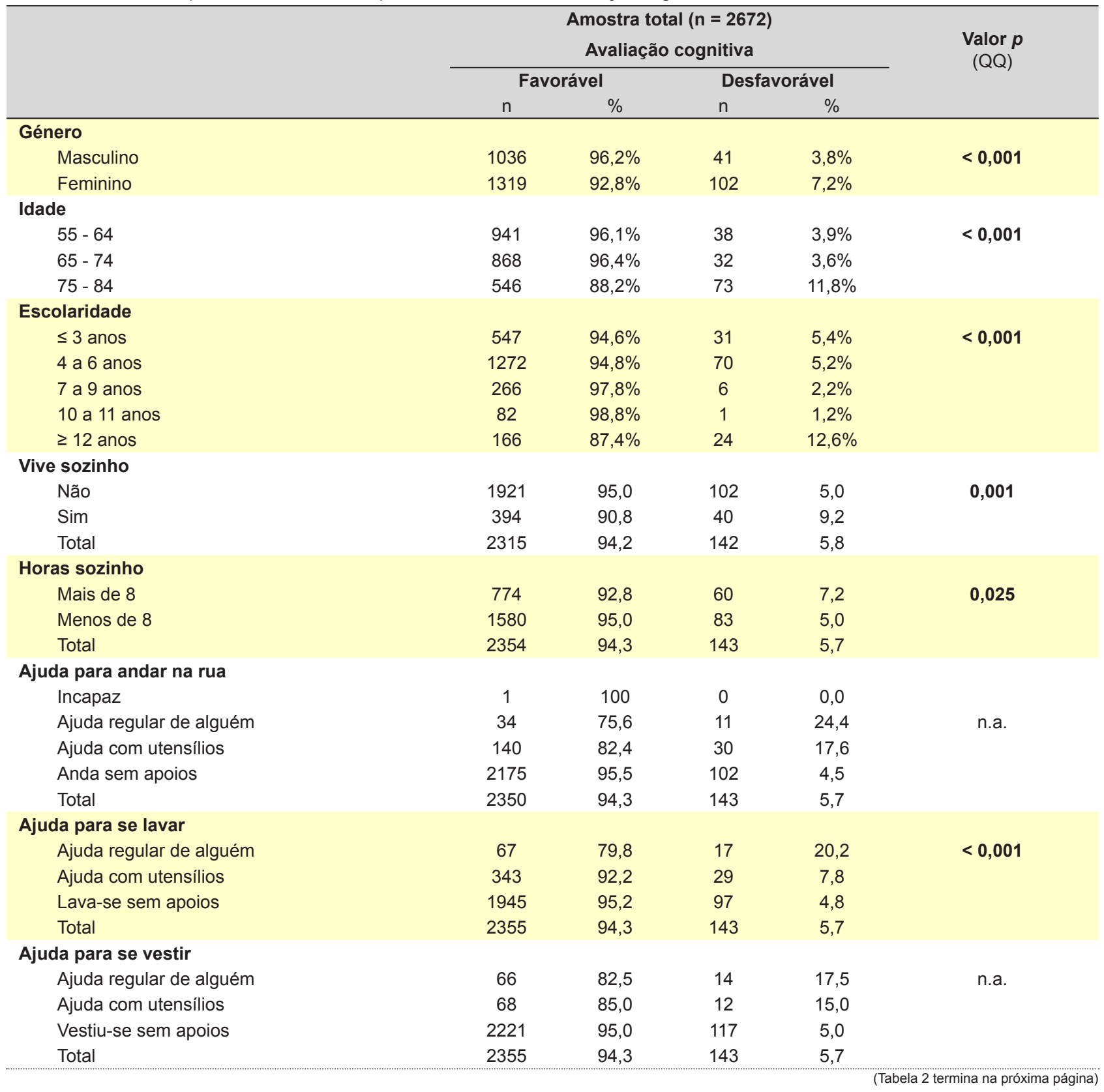


cada associação. Os resultados encontram-se descritos na Tabela 3.

Verificámos uma associação estatisticamente significativa entre a avaliação cognitiva e o facto de a pessoa viver sozinha, o tempo que vive sozinha (OR = 1,476; IC 95\%: $1,047-2,080)$, a autonomia a andar na rua (OR $=4,996$; IC 95\%: 3,370 - 7,407), lavar-se (OR = 2,250; IC 95\%: 1,559 - 3,246), vestir-se (OR = 3,683; IC 95\%: 2,327 - 5,831), comer $(\mathrm{OR}=2,471$; IC 95\%: 1,442 - 4,234), preparar refeições (OR = 1,833; IC 95\%: 1,301 -2,581), fazer compras (OR = 2,500; IC 95\%: 1,779 - 3,515), gerir dinheiro (OR = 2,993; IC 95\%: 2,129 - 4,207) e tomar medicamentos (OR = 3,450; IC 95\%: 2,353 - 5,060).

A partir destes dados verifica-se que viver sozinho está estatisticamente relacionado com uma avaliação cognitiva desfavorável. Também se verifica que a autonomia em grande parte das atividades analisadas se associa a uma avaliação cognitiva favorável. A variável que mostra uma magnitude de associação mais forte é a autonomia a andar na rua, em que indivíduos autónomos têm uma maior probabilidade $(\mathrm{OR}=4,996)$ de obter um resultado favorável em relação a não autónomos.

A autonomia a lavar a roupa não se mostrou estatisticamente relacionada com a avaliação cognitiva.

Foi efetuada uma análise de regressão logística com todas as variáveis que mostraram uma associação significativa com o score obtido na avaliação cognitiva. O modelo final obtido encontra-se descrito na Tabela 4.

A variável com maior probabilidade de conduzir a uma avaliação cognitiva favorável é a autonomia a andar na rua, em que os indivíduos autónomos mostram ter 3,196 vezes a probabilidade de obter um score favorável, em

Tabela 2 - Análise comparativa de variáveis capazes de influenciar a avaliação cognitiva (continuação)

\begin{tabular}{|c|c|c|c|c|c|}
\hline & \multicolumn{4}{|c|}{ Amostra total $(n=2672)$} & \multirow{4}{*}{$\begin{array}{c}\text { Valor } p \\
(Q Q)\end{array}$} \\
\hline & \multicolumn{4}{|c|}{ Avaliação cognitiva } & \\
\hline & \multicolumn{2}{|c|}{ Favorável } & \multicolumn{2}{|c|}{ Desfavorável } & \\
\hline & $\mathrm{n}$ & $\%$ & $\mathrm{n}$ & $\%$ & \\
\hline \multicolumn{6}{|l|}{ Ajuda para comer } \\
\hline Incapaz & 5 & 100 & 0 & 0,0 & \\
\hline Ajuda regular de alguém & 6 & 54,5 & 5 & 45,5 & n.a. \\
\hline Ajuda com utensílios & 112 & 90,3 & 12 & 9,7 & \\
\hline Alimenta-se sozinho & 2217 & 94,7 & 124 & 5,3 & \\
\hline Total & 2340 & 94,3 & 141 & 5,7 & \\
\hline \multicolumn{6}{|l|}{ Ajuda para preparar refeições } \\
\hline Incapaz & 361 & 89,8 & 41 & 10,2 & \\
\hline Colaboração parcial & 293 & 94,5 & 17 & 5,5 & $<0,001$ \\
\hline Preparação desadequada & 38 & 90,5 & 4 & 9,5 & \\
\hline Preparação adequada & 1657 & 95,3 & 81 & 4,7 & \\
\hline Total & 2349 & 94,3 & 143 & 5,7 & \\
\hline \multicolumn{6}{|l|}{ Ajuda para fazer compras } \\
\hline Incapaz & 125 & 81,2 & 29 & 18,8 & \\
\hline Compras levadas a casa & 43 & 84,3 & 8 & 15,7 & $<0,001$ \\
\hline Compras pequenas ou acompanhado & 610 & 93,6 & 42 & 6,4 & \\
\hline Compras feitas sozinho & 1576 & 96,1 & 64 & 3,9 & \\
\hline Total & 2354 & 94,3 & 143 & 5,7 & \\
\hline \multicolumn{6}{|l|}{ Ajuda para fazer gerir dinheiro } \\
\hline Incapaz & 38 & 58,5 & 27 & 41,5 & \\
\hline Regularmente & 88 & 83,3 & 17 & 16,2 & $<0,001$ \\
\hline Ocasionalmente & 495 & 94,3 & 30 & 5,7 & \\
\hline Lidou sozinho & 1733 & 96,2 & 69 & 3,8 & \\
\hline Total & 2354 & 94,3 & 143 & 5,7 & \\
\hline \multicolumn{6}{|l|}{ Ajuda para tomar medicamentos } \\
\hline Incapaz & 8 & 50,0 & 8 & 50,0 & \\
\hline Regularmente & 48 & 75,0 & 16 & 25,0 & n.a. \\
\hline Ocasionalmente & 196 & 91,6 & 18 & 8,4 & \\
\hline Tomou na dose e tempo certos & 2091 & 95,4 & 101 & 4,6 & \\
\hline Total & 2343 & 94,2 & 143 & 5,8 & \\
\hline \multicolumn{6}{|l|}{ Ajuda para lavar/tratar a roupa } \\
\hline Incapaz & 583 & 92,0 & 51 & 8,0 & \\
\hline Regularmente em todas as peças & 309 & 94,5 & 18 & 5,5 & 0,028 \\
\hline Regularmente em peças grandes & 179 & 96,2 & 7 & 3,8 & \\
\hline Tratou sozinho da roupa & 1281 & 95,0 & 67 & 5,0 & \\
\hline Total & 2352 & 94,3 & 143 & 5,7 & \\
\hline
\end{tabular}

QQ: Teste do qui-quadrado; n.a.: Não aplicável. Percentagem calculada em linha 
comparação com os não autónomos (IC 95\%: 2,005 $5,092)$. Segue-se em ordem decrescente de magnitude o ter autonomia para: preparar refeições $(\mathrm{OR}=2,796$; IC 95\%: 1,715 - 4,560); gerir dinheiro $(\mathrm{OR}=2,163$; IC 95\%: 1,422 - 3,290); tomar medicamentos (OR $=1,927$; IC 95\%: $1,211-3,065)$.

Os indivíduos do género feminino $(\mathrm{OR}=0,333$; IC $95 \%$ : $0,200-0,554)$, os indivíduos com idade igual ou superior a 75 anos (OR = 0,540; IC 95\%: 0,332-0,877), e os indi- víduos com uma escolaridade de 4 a 6 anos (OR $=0,557$; IC 95\%: 0,343 - 0,905) ou $\geq 12$ anos (OR = 0,145; IC 95\%: 0,075 - 0,280) mostram um maior risco de ter uma avaliação cognitiva desfavorável.

\section{DISCUSSÃO}

O enriquecimento intelectual ao longo da vida, separando as duas componentes, escolaridade e atividade cognitiva em indivíduos com idade igual ou superior a 55 anos,

Tabela 3 - Análise comparativa de variáveis capazes de influenciar a avaliação cognitiva com agregação de categorias

\begin{tabular}{|c|c|c|c|c|c|c|c|}
\hline \multicolumn{8}{|c|}{ Amostra total $(n=2672)$} \\
\hline & \multicolumn{4}{|c|}{ Avaliação cognitiva } & \multirow{3}{*}{$\begin{array}{c}\text { Valor } p \\
\text { (QQ) }\end{array}$} & \multirow{3}{*}{ OR } & \multirow[t]{3}{*}{ OR (IC 95\%) } \\
\hline & \multicolumn{2}{|c|}{ Favorável (1) } & \multicolumn{2}{|c|}{ Desfavorável (0) } & & & \\
\hline & $\mathrm{n}$ & $\%$ & $\mathrm{n}$ & $\%$ & & & \\
\hline \multicolumn{8}{|l|}{ Vive sozinho } \\
\hline Não - classe de referência & 1921 & 95,0 & 102 & 5,0 & 0,001 & 1 & \\
\hline $\operatorname{Sim}$ & 394 & 90,8 & 40 & 9,2 & & 0,523 & $(0,357-0,766)$ \\
\hline Total & 2315 & 94,2 & 142 & 5,8 & & & \\
\hline \multicolumn{8}{|l|}{ Horas sozinho } \\
\hline 8 horas ou mais - classe de referência & 774 & 92,8 & 60 & 7,2 & 0,025 & 1 & \\
\hline Menos de 8 & 1580 & 95,0 & 83 & 5,0 & & 1,476 & $(1,047-2,080)$ \\
\hline Total & 2354 & 94,3 & 143 & 5,7 & & & \\
\hline \multicolumn{8}{|l|}{ Autonomia a andar na rua } \\
\hline Não - classe de referência & 175 & 81,0 & 41 & 19,0 & $<0,001$ & 1 & \\
\hline Sim & 2175 & 95,5 & 102 & 4,5 & & 4,996 & $(3,370-7,407)$ \\
\hline Total & 2350 & 94,3 & 143 & 5,7 & & & \\
\hline \multicolumn{8}{|l|}{ Autonomia a lavar-se } \\
\hline Não - classe de referência & 410 & 89,9 & 46 & 10,1 & $<0,001$ & 1 & \\
\hline $\operatorname{Sim}$ & 1945 & 95,2 & 97 & 4,8 & & 2,250 & $(1,559-3,246)$ \\
\hline Total & 2355 & 94,3 & 143 & 5,7 & & & \\
\hline \multicolumn{8}{|l|}{ Autonomia a vestir-se } \\
\hline Não - classe de referência & 134 & 83,8 & 26 & 16,2 & $<0,001$ & 1 & \\
\hline $\operatorname{Sim}$ & 2221 & 95,0 & 117 & 5,0 & & 3,683 & $(2,327-5,831)$ \\
\hline Total & 2355 & 94,3 & 143 & 5,7 & & & \\
\hline \multicolumn{8}{|l|}{ Autonomia a comer } \\
\hline Não - classe de referência & 123 & 87,9 & 17 & 12,1 & 0,001 & 1 & \\
\hline $\operatorname{Sim}$ & 2217 & 94,7 & 124 & 5,3 & & 2,471 & $(1,442-4,234)$ \\
\hline Total & 2340 & 94,3 & 141 & 5,7 & & & \\
\hline \multicolumn{8}{|l|}{ Autonomia a preparar refeições } \\
\hline Não - classe de referência & 692 & 91,8 & 62 & 8,2 & $<0,001$ & 1 & \\
\hline $\operatorname{Sim}$ & 1657 & 95,3 & 81 & 4,7 & & 1,833 & $(1,301-2,581)$ \\
\hline Total & 2349 & 94,3 & 143 & 5,7 & & & \\
\hline \multicolumn{8}{|l|}{ Autonomia a fazer compras } \\
\hline Não - classe de referência & 778 & 90,8 & 79 & 9,2 & $<0,001$ & 1 & \\
\hline $\operatorname{Sim}$ & 1576 & 96,1 & 64 & 3,9 & & 2,500 & $(1,779-3,515)$ \\
\hline Total & 2354 & 94,3 & 143 & 5,7 & & & \\
\hline \multicolumn{8}{|l|}{ Autonomia a gerir dinheiro } \\
\hline Não - classe de referência & 621 & 89,4 & 74 & 10,6 & $<0,001$ & 1 & \\
\hline $\operatorname{Sim}$ & 1733 & 96,2 & 69 & 3,8 & & 2,993 & $(2,129-4,207)$ \\
\hline Total & 2354 & 94,3 & 143 & 5,7 & & & \\
\hline \multicolumn{8}{|l|}{ Autonomia a tomar medicamentos } \\
\hline Não - classe de referência & 252 & 85,7 & 42 & 14,3 & $<0,001$ & 1 & \\
\hline $\operatorname{Sim}$ & 2091 & 95,4 & 101 & 4,6 & & 3,450 & $(2,353-5,060)$ \\
\hline Total & 2343 & 94,2 & 143 & 5,7 & & & \\
\hline \multicolumn{8}{|l|}{ Autonomia a lavar a roupa } \\
\hline Não - classe de referência & 1071 & 93,4 & 76 & 6,6 & 0,076 & 1 & \\
\hline $\operatorname{Sim}$ & 1281 & 95,0 & 67 & 5,0 & & 1,357 & $(0,967-1,903)$ \\
\hline Total & 2352 & 94,3 & 143 & 5,7 & & & \\
\hline
\end{tabular}

QQ: Teste do qui-quadrado; OR: odds ratio. Percentagem calculada em linha. OR foi determinado face à condição favorável. 
mostrou que o efeito benéfico da estimulação da atividade cognitiva na linha basal não era muito influenciado pelo aumento do número de anos de escolaridade. Em geral, níveis mais altos de educação estão associados com os níveis mais altos de cognição. No entanto, como se observa na Tabela 4, esta relação não é linear e os extremos de escolaridade evidenciam uma avaliação cognitiva desfavorável, o que sugere que a estimulação cognitiva pode ter efeitos diferentes, consoante a idade em que é feita. Tem sido referido, por alguns autores, que uma estimulação cognitiva em idades menos avançadas (entre os 55 e os 64 anos) se associa a uma menor deterioração cognitiva. Verificou-se também, que a atividade cognitiva se mantem preservada durante mais tempo em indivíduos com maior número de anos de escolaridade. ${ }^{17}$

O Estudo EPEPP demonstrou que o número de horas de isolamento está negativamente associado ao estado cognitivo. A falta de uma rede social de suporte bem estruturada aumenta a fragilidade do idoso, que pode ser acentuada pela combinação do declínio físico, com o compromisso da mobilidade e com o declínio cognitivo associados à idade. Para um envelhecimento bem-sucedido, uma premissa fundamental é o envolvimento do idoso em atividades intelectuais, sociais e fisicamente estimulantes. ${ }^{18-20}$

O estudo univariado realizado mostra-nos que todas as variáveis pesquisadas estão significativamente associadas com uma melhor avaliação cognitiva (exceto viver sozinho e autonomia a lavar a roupa).

Os dados da literatura evidenciam que a falta de interação social está associada a um aumento de incidência de demência, sendo comparável com outros fatores de risco já estabelecidos incluindo um baixo nível de escolaridade, inatividade física e depressão. ${ }^{21}$

O número de horas que o indivíduo passa sozinho está muitas vezes associado à solidão, entendida como uma experiência desagradável que ocorre maioritariamente quando a rede social da pessoa é considerada deficiente. Segundo alguns autores, nos indivíduos que estão sós o risco de desenvolver demência, em particular doença de Alzheimer, duplica e em geral o declínio cognitivo é mais rápido do que o observado nos indivíduos que têm uma rede social de suporte. ${ }^{18}$ Como tal, a solidão, neste estudo assumida através da objetivação do tempo que o idoso passa sozinho, é uma forma indireta de avaliar subjetivamente a qualidade do suporte social. Um bom desempenho das AVDsI e o apoio emocional reduzem a solidão, preservando o funcionamento cognitivo. Este efeito protetor do apoio emocional tem sido demonstrado ser mais forte entre os adultos com 65 ou mais anos. ${ }^{18} \mathrm{~A}$ inatividade social e a solidão, independentemente ou em combinação, são

Tabela 4 - Análise de regressão logística múltipla para avaliação cognitiva favorável

\begin{tabular}{|c|c|c|c|c|}
\hline & & OR & ICI 95\% & Valor $p$ \\
\hline \multicolumn{5}{|l|}{ Género } \\
\hline Masculino - classe de referência & & 1 & & \\
\hline Feminino & & 0,333 & $0,200-0,554$ & $<0,001$ \\
\hline \multicolumn{5}{|l|}{ Idade } \\
\hline 55 - 64 - classe de referência & & 1 & & \\
\hline $65-74$ & & 1,298 & $0,779-2,164$ & 0,317 \\
\hline $75-84$ & & 0,540 & $0,332-0,877$ & 0,013 \\
\hline \multicolumn{5}{|l|}{ Escolaridade } \\
\hline$\leq 3$ anos - classe de referência & & 1 & & \\
\hline 4 a 6 anos & & 0,557 & $0,343-0,905$ & 0,018 \\
\hline 7 a 9 anos & & 1,290 & $0,510-3,266$ & 0,590 \\
\hline 10 a 11 anos & & 1,370 & $0,178-10,523$ & 0,762 \\
\hline$\geq 12$ anos & & 0,145 & $0,075-0,280$ & $<0,001$ \\
\hline \multicolumn{5}{|l|}{ Pessoa vive sozinha } \\
\hline Não - classe de referência & & 1 & - & - \\
\hline Sim & & 0,662 & $0,412-1,064$ & 0,089 \\
\hline \multicolumn{5}{|l|}{ Autonomia a andar na rua } \\
\hline Não - classe de referência & & 1 & - & - \\
\hline Sim & & 3,196 & $2,005-5,092$ & $<0,001$ \\
\hline \multicolumn{5}{|l|}{ Autonomia a preparar refeições } \\
\hline Não - classe de referência & & 1 & - & - \\
\hline Sim & & 2,796 & $1,715-4,560$ & $<0,001$ \\
\hline \multicolumn{5}{|l|}{ Autonomia a gerir dinheiro } \\
\hline Não - classe de referência & & 1 & - & - \\
\hline $\operatorname{Sim}$ & & 2,163 & $1,422-3,290$ & $<0,001$ \\
\hline \multicolumn{5}{|l|}{ Autonomia a tomar medicamentos } \\
\hline Não - classe de referência & & 1 & - & - \\
\hline $\operatorname{Sim}$ & & 1,927 & $1,211-3,065$ & 0,006 \\
\hline Valor $p$ do modelo & $<0,001$ & & & \\
\hline Teste de Hosmer-Lemeshow & 0,723 & & & \\
\hline Taxa de validade do modelo & 94,5 & & & \\
\hline
\end{tabular}


indicadores de risco de mortalidade numa população idosa. ${ }^{22,23}$

O Estudo EPEPP revelou que a autonomia física para tarefas relacionadas com o quotidiano, tais como o andar na rua, lavar-se, vestir-se, comer, preparar refeições, fazer compras, gerir dinheiro, tomar medicamentos e lavar/ tratar a roupa, se associam a uma melhor avaliação cognitiva. Como vimos anteriormente, esta é influenciada negativamente pelo número de horas que um indivíduo vive sozinho. Estas correlações são reforçadas quando se faz a análise com agregação por categorias das variáveis em estudo, particularmente no que diz respeito à autonomia em andar na rua.

Os dados obtidos permitem estimar a associação expurgada de multicolineridade entre a variável dependente, cognição, e as variáveis consideradas preditoras incluídas neste estudo, nomeadamente, autonomia a andar na rua, gerir dinheiro, tomar medicamentos e preparar refeições. As limitações da metodologia utilizada neste estudo (estudo transversal, cross-sectional) apenas permitem afirmar, à semelhança do que referem outros autores, que um maior envolvimento nas AVDs está associado a um menor declínio cognitivo. ${ }^{24-27}$

A autonomia do indivíduo para realizar tarefas da vida diária, pode tornar-se uma ferramenta poderosa na prevenção, não só do comprometimento cognitivo, mas também de patologias neurodegenerativas para as quais o envelhecimento é um fator de risco, tais como a doença de Alzheimer, a forma mais comum de demência no idoso. A autonomia física e cognitiva, associada com o estilo de vida e atividades quotidianas, têm também um efeito protetor da patologia cerebrovascular. ${ }^{28-34} \mathrm{~A}$ cognição contextualizada em sentido lato, condiciona a interação do indivíduo com o ambiente que o rodeia, quer a nível físico quer social. Ou seja, a cognição e a autonomia do indivíduo estão interligadas e não podem ser separadas da condição social e do meio onde está inserido. ${ }^{35}$

\section{CONCLUSÃO}

A associação do número de horas que o indivíduo passa sozinho com os resultados adversos para a saúde, observada neste estudo, deve ser um alerta para a instituição de políticas de saúde que contemplem a redução do isolamento social, melhorando as relações intergeracionais, e as redes sociais dos grupos de idosos mais vulneráveis.

A preservação da autonomia física e do estado cognitivo são determinantes para a manutenção da autonomia nas atividades de vida diária, que requerem capacidade de planear e realizar tarefas e, consequentemente, são uma componente chave para um envelhecimento de sucesso. A deteção precoce de dificuldades funcionais permitirá delinear exercícios físicos compensatórios, de acordo com a capacidade física do indivíduo, que moderem a perda da autonomia física e indiretamente o declínio cognitivo na população idosa.

Como já foi referido este estudo tem duas limitações, por um lado incide num coorte de voluntários saudáveis sem incapacidade física que os impedisse de se deslocar ao local de entrevista e, por outro lado foram aplicadas versões simplificadas dos inquéritos de avaliação funcional dos idosos, validadas por teste re-teste numa amostra reduzida da população em estudo. No entanto, a dimensão da amostra estudada, representativa da população portuguesa, mostra a importância da colheita de informação sistematizada junto dos idosos e seus familiares, ou daqueles que com eles privam, quando referente a atividades de vida diária, atendendo à sua relação com a função cognitiva.

Em conclusão, para um envelhecimento ativo e saudável é determinante a manutenção de uma rede social ativa. A deteção precoce de um declínio na autonomia física e instrumental, avaliada pela capacidade de execução de atividades de vida diária, faz-se acompanhar, com frequência, de perturbação da cognição, podendo servir de indicador da necessidade de implementar medidas que promovam uma boa autonomia no processo do envelhecimento.

\section{AGRADECIMENTOS}

Os autores gostariam de agradecer a Leonor Salguinho pelo trabalho de edição em termos bibliográficos.

\section{PROTECÇÃO DE PESSOAS E CONFIDENCIALIDADE DOS DADOS}

Este estudo foi aprovado pela Comissão de Ética da Faculdade de Medicina da Universidade de Coimbra. Os dados que pudessem permitir a identificação de qualquer participante foram codificados antes do manuseamento da informação e não foram registados ou tratados dados que permitissem a identificação pessoal.

\section{CONFLITOS DE INTERESSE}

Todos os autores declaram que participaram no trabaIho, se responsabilizam por ele e que não existe, da parte de qualquer dos autores conflito de interesses nas afirmações proferidas no trabalho.

\section{FONTES DE FINANCIAMENTO}

Saúde XXI - Programa Operacional da Saúde (Ministério da Saúde).

\section{REFERÊNCIAS}

1. Instituto Nacional de Estatística. Projeções de população residente 2012-2060. Lisboa: INE; 2014.

2. Christensen K, Doblhammer G, Rau R, Vaupel JW. Ageing populations: the challenges ahead. Lancet. 2009;374:1196-208.

3. Suzman R, Beard JR, Boerma T, Chatterji S. Health in an ageing world - what do we know? Lancet. 2015;385:484-6.

4. Bherer L. Cognitive plasticity in older adults: effects of cognitive training

and physical exercise. Ann N Y Acad Sci. 2015;1337:1-6.

5. Perneczky R, Pohl C, Sorg C, Hartmann J, Komossa K, Alexopoulos $P$, et al. Complex activities of daily living in mild cognitive impairment: conceptual and diagnostic issues. Age Ageing. 2006;35:240-5.

6. Royall DR. Mild cognitive impairment and functional status. J Am Geriatr Soc. 2006;54:163-5.

7. De Vriendt P, Gorus E, Cornelis E, Bautmans I, Petrovic M, Mets T. The 
advanced activities of daily living: a tool allowing the evaluation of subtle functional decline in mild cognitive impairment. J Nutr Health Aging. 2013;17:64-71.

8. de Paula JJ, Bertola L, de Ávila RT, Assis LO, Albuquerque M, Bicalho MA, et al. Development, validity, and reliability of the General Activities of Daily Living Scale: a multidimensional measure of activities of daily living for older people. Rev Bras Psiquiat. 2014;36:143-52.

9. Kamo T, Nishida Y. Direct and indirect effects of nutritional status, physical function and cognitive function on activities of daily living in Japanese older adults requiring long-term care. Geriatr Gerontol Int. 2014;14:799-805.

10. Stessman J, Rottenberg Y, Shimshilashvili I, Ein-Mor E, Jacobs JM. Loneliness, health, and longevity. J Gerontol A Biol Sci Med Sci. 2014;69:744-50.

11. Miller DI, Taler V, Davidson PS, Messier C. Measuring the impact of exercise on cognitive aging: methodological issues. Neurobiol Aging. 2012;33:622.e29-43.

12. Berr C, Balard F, Blain H, Robine JM. Vieillissement, l'émergence d'une nouvelle population Med Sci. 2012;28:281-7.

13. Leist AK, Hessel P, Avendano M. Do economic recessions during early and mid-adulthood influence cognitive function in older age? J Epidemiol Community Health. 2014;68:151-8.

14. Resende de Oliveira C, Santos Rosa M, Mota Pinto A, Silveira Botelho MA, Morais A, Teixeira Veríssimo M. Estudo do Perfil do Envelhecimento da População Portuguesa - EPEPP. Coimbra: Faculdade de Medicina, Universidade de Coimbra; 2008

15. Botelho MA. Autonomia funcional em idosos: caracterização multidimensional em idosos utentes de um centro de saúde urbano. Porto: Laboratórios Bial; 2000.

16. Rodrigues V, Mota-Pinto A, de Sousa B, Botelho A, Alves C, Resende de Oliveira $C$. The aging profile of the portuguese population: a principal component analysis. J Community Health. 2014;39:747-52.

17. Vemuri P, Lesnick TG, Przybelski SA, Machulda M, Knopman DS, Mielke $\mathrm{MM}$, et al. Association of lifetime intellectual enrichment with cognitive decline in the older population. JAMA Neurol. 2014;71:1017-24.

18. Ellwardt L, Aartsen M, Deeg D, Steverink N. Does loneliness mediate the relation between social support and cognitive functioning in later life? Soc Sci Med. 2013;98:116-24.

19. Yen IH, Michael YL, Perdue L. Neighbourhood environment in studies of health of older adults: a systematic review. Am J Prev Med. 2009;37:45563.

20. Windsor TD, Gerstorf D, Pearson E, Ryan LH, Anstey KJ. Positive and negative social exchanges and cognitive aging in young-old adults: Differential associations across family, friend, and spouse domains. Psychol Aging. 2014;29:28-43.

21. Kuiper JS, Zuidersma M, Voshaar RC, Zuidema SU, van den Heuvel ER, Stolk RP, et al. Social relationships and risk of dementia: A systematic review and meta-analysis of longitudinal cohort studies. Ageing Res Rev. 2015;22:39-57.

22. Tilvis RS, Routasalo $P$, Karppinen $H$, Strandberg TE, Kautiainen $H$, Pitkala $\mathrm{KH}$. Social isolation, social activity and loneliness as survival indicators in old age; a nationwide survey with a 7-year follow-up. Eur Geriatr Med. 2011;3:18-22

23. Mota-Pinto A, Rodrigues $\mathrm{V}$, Botelho A, Teixeira-Veríssimo M, Morais A, Alves $C$, et al. A sociodemographic study of aging in the portuguese population: the EPEPP study. Arch Gerontol Geriatrics. 2011;52:304-8.

24. Ghisletta P, Bickel JF, Lövdén M. Does activity engagement protect against cognitive decline in old age? Methodological and analytical considerations. J Gerontol B Psychol Sci Soc Sci. 2006;61:253-61.

25. Buchman AS, Wilson RS, Yu L, James BD, Boyle PA, Bennett DA. Total daily activity declines more rapidly with increasing age in older adults Arch Gerontol Geriatr. 2014;58:74-9.

26. Jiang $\mathrm{C}, \mathrm{Xu} \mathrm{Y}$. The association between mild cognitive impairment and doing housework. Aging Mental Health. 2014;18:212-6.

27. Schmitter-Edgecombe M, Parsey C, Lamb R. Development and psychometric properties of the instrumental activities of daily living: compensation scale. Arch Clin Neuropsychol. 2014;29:776-92.

28. Lucas SJ, Ainslie PN, Murrell CJ, Thomas KN, Franz EA, Cotter JD. Effect of age on exercise-induced alterations in cognitive executive function: relationship to cerebral perfusion. Exp Gerontol. 2012;47:54151

29. Wirth M, Haase CM, Villeneuve S, Vogel J, Jagust WJ. Neuroprotective pathways: lifestyle activity, brain pathology, and cognition in cognitively normal older adults. Neurobiol Aging. 2014;35:1873-82.

30. Gow AJ, Bastin ME, Munoz Maniega S, Valdes Hernandez MC, Morris $Z$, Murray C, et al. Neuroprotective lifestyles and the aging brain: activity, atrophy, and white matter integrity. Neurology. 2012;79:1802-8.

31. Lovden M, Bodammer NC, Kuhn S, Kaufmann J, Schutze H, Tempelmann C, et al. Experiencedependent plasticity of white-matter microstructure extends into old age. Neuropsychologia. 2010;48:387883.

32. Erickson KI, Voss MW, Prakash RS, Basak C, Szabo A, Chaddock L, et al. Exercise training increases size of hippocampus and improves memory. Proc Natl Acad Sci USA. 2011;108:3017-22.

33. Floel A, Ruscheweyh R, Kruger K, Willemer C, Winter B, Volker K, et al. Physical activity andmemory functions: are neurotrophins and cerebral gray matter volume the missing link? Neuroimage. 2010;49:2756-63.

34. Gons RA, Tuladhar AM, de Laat KF, van Norden AG, van Dijk EJ, Norris $D G$, et al. Physical activity is related to the structural integrity of cerebral white matter. Neurology. 2013;81:971-76.

35. Durning SJ, Artino AR, Holmboe E, Beckman TJ, van der Vleuten C, Schuwirth L. Aging and cognitive performance: Challenges and implications for physicians practicing in the 21st century. J Contin Educ Health Prof. 2010;30:153-60. 